\title{
Noise Control Transmission Methods of the Combustion Engine by Means of Reduction of the Vibration
}

\author{
Stanislav ŽIARAN, Ondrej CHLEBO \\ Faculty of Mechanical Engineering \\ Slovak University of Technology in Bratislava \\ Nam. slobody 17, 81231 Bratislava, Slovakia; e-mail: \{stanislav.ziaran, ondrej.chlebo\}@stuba.sk
}

(received March 11, 2016; accepted March 30, 2016)

\begin{abstract}
Transmission of vibroacoustic energy from an internal combustion engine (ICE) to its surroundings largely depends on how it is mounted, on available transmission paths and on the construction of the vehicle body and/or its surrounding structures. This is especially true in low speed engines in enclosed areas which generate perceptually weak noise, but strong low-frequency waves which energy has a negative impact on human health, comfort and driving safety especially in prolonged exposure to the source. The primary aim of the article was to analyse components of the ICE unit which had a determining impact on the reduction of low-frequency waves. Thus, the structurally transmitted noise from the ICE to its surrounding structure (body of the passenger vehicle) was analysed. The results of the vibroacoustic measurements were compared to modal analysis in order to determine possible resonance sources in the vehicle body and/or for assessing the influence of the vehicles safety gear on the generated vibroacoustic energy transfer into the cabin area of the passenger vehicle. Measurements were made for a passenger vehicle at rest and operating in its most common operational speed as well as for the stationary ICE of a cogenerate unit (CGU). Measurements and FFT analysis were used for the detection of the vibroacoustic energy sound pressure level (noise) and mechanical vibration. Firstly, the low-frequency noise sources were determined and their direct effects on the human body were investigated. Finally, this paper suggests some measures which may contribute to the reduction of undesirable vibroacoustic energy in enclosed areas.
\end{abstract}

Keywords: combustion engine; low-frequency waves; transmission; health; measures.

\section{Introduction}

Various types of passive vibration isolators are typically used to reduce the transmission of vibrations. Examples include automobile engine mounts, resilient supports for cogeneration units and buildings, resilient mounts and flexible shaft couplings for shipboard machinery, and small isolators in household appliances. Isolation and structural damping constitute the two most widely applicable means for the control of vibration or structure-borne sound, particularly in the audible frequency range. In essence, vibration isolation involves the use of a resilient connection between a source of vibration and the item to be isolated, so that this item vibrates less than it would, if a rigid connection were to be used. In a typical situation, the source consists of a vibrating machine or structure and the item to be protected is the human body, environment, instruments, machines etc. Many salient features of vibration isolation can be analysed in terms of a simple model consisting of a rigid mass that is connected to a support via a vibroisolator (resilient element) and that is constrained to translate along a single axis (ZIARAN, 2006; 2010). More complex models are needed to address situations where the magnitude of excitation depends on the motions, where an additional vibroisolator-mass system is inserted between the primary system and its support, and/or at comparatively high frequencies where the vibroisolator mass plays a significant role or where the isolated items do not behave as rigid masses. Other complications arise because of non-uniaxial motions and nonlinearities.

\section{Goals, object of the investigation, instrumentation and methodology}

The goals of the study were to investigate and analyze the flow of structure-borne vibroacoustic energy 
of a deterministic excitation and its effects on neighbouring structures such as the cabin of a vehicle or enclosed areas where cogenerate units (CGU) exist. The study shows that the low-frequency excitation waves can also cause a resonance state at higher frequencies, which can negatively influence the dynamic loading of the surrounding structures resulting in a high level of noise with respect to their surroundings. The frequency spectrum of the measured signal and Eigen (natural) frequencies of the Eigen modes are also compared and analysed for the investigated objects. This paper mentions energy flow from the source (mounting) into surroundings.

The measurement of structurally-borne vibroacoustic energy was performed on a passenger vehicle at predetermined operational conditions in order to investigate the transmission of energy noise from the drivinggear to the surrounding components of the vehicles body, particularly roof, doors, and floor pan (ORESKY et al., 2012; ZIARAN, 2016). The same investigation was performed on a stationary CGU and its impact on the surrounding area. For the vehicle, the primary source of vibration and noise was a gasoline powered ICE running closely to frequent speed $4000 \mathrm{rpm}$ and gas ICE running to speed $1500 \mathrm{rpm}$. The vehicle was stationary during measurements.

The deterministic signal was generated by the ICE and the response on the vehicle body and surrounding structures was performed using the FFT analyzer PULSE Bruel \& Kjaer platform. This portable analyzer represents a system which guarantees reliable measurement processes, analysis, and evaluation. The system consists of a piezoelectric accelerometer with a frequency range from $1 \mathrm{~Hz}$ to $10 \mathrm{kHz}$ (amplitude $\pm 10 \%$ ), modal hammer, and display and memory module. To identify the energy dominant Eigen frequencies more precisely, the fast Fourier transform (FFT) analysis was carried out using the FFT analyzer PULSE. The methodology presented in the article can also be applied to other excitation sources of low frequency vibration. Sensor mounting on the structural elements of the investigated objects was consistent with ISO 5348 requirements for accelerometers and with respect to past experience (ZIARAN, 2006; 2013c; 2016). The goal was to ensure that the sensor (acceleration, force) correctly reproduces the motion of the analysed component without interfering with its response. The sound pressure level was analyzed using Sound Level Meters Bruel \& Kjaer 2250 platform.

Other than the frequency range, for the type of signal, it is also very important to select the appropriate type of averaging, number of averages per unit time, as well as a suitable time window (ZIARAN, 2013c).

The measurement of the transmission loss assumes that the material behaves in a linear way and that it has negligible mass compared with the mass loading. The methodology determines the impedance of the ma- terial when loaded by a mass, providing a compressive force equivalent to that found when the resilient material (element) was placed between the ICE unit and vehicle structure or between the support frame of the CGU and its foundation. This was done by measuring the transfer function of the mass-loaded material at all required frequencies (ZIARAN, 2010; ISO 10846-1). The methodology utilizes a vibration excitation system with a resilient element (material) placed at the bottom of the system (Fig. 1). Two accelerometers measure the vibration on the coupling flange of a vibration generator (in the presented case on the sub-frame of the ICE and the engine of the CGU), $a_{1}$, and the vibration of the coupling flange mass $m$ (the vehicle body and the frame of the CGU), $a_{2}$. Assuming the test resilient element - flexible part of coupling (e.g. silentblock) has negligible mass, the equation of motion is

$$
m \ddot{x}_{2}=-Z_{\mathrm{m}}\left(\dot{x}_{2}-\dot{x}_{1}\right)
$$

from which the mechanical impedance of the resilient element is

$$
Z_{\mathrm{m}}=\frac{j \omega m}{\left[\frac{A_{1}}{A_{2}}\right]-1}
$$

at a single frequency it follows that (ZIARAN, 2010)

$$
\frac{A_{1}(\mathrm{j} \omega)}{A_{2}(\mathrm{j} \omega)}=\text { magnitude } \times[\cos (\text { phase })+\mathrm{j} \sin (\text { phase })],
$$

where $A_{i}(\mathrm{j} \omega)$ is Fourier's transformation of acceleration $a_{i}$; magnitude - the magnitude of the ratio denoted by $A_{1} / A_{2}$; phase - the phase difference between $A_{1}$ and $A_{2}$.

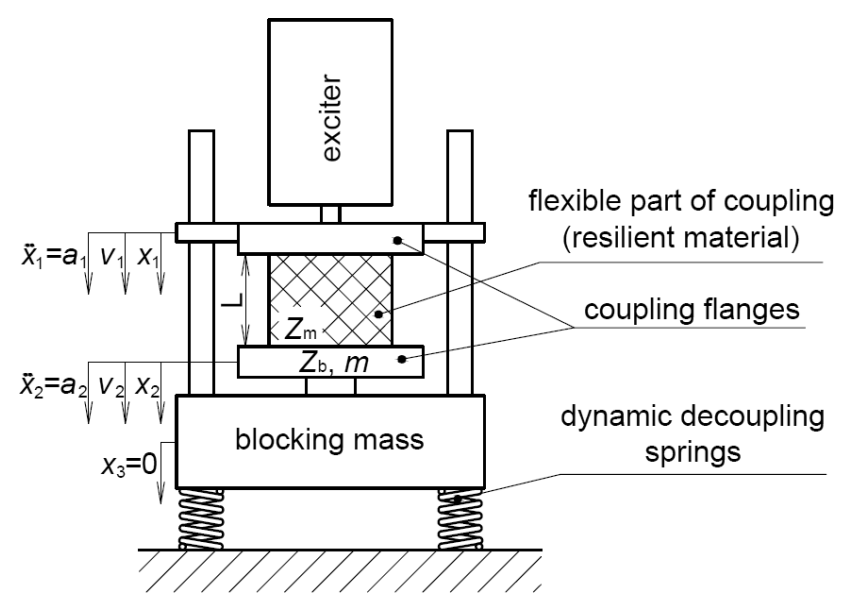

Fig. 1. Theoretical model for the determination of the transfer function and/or transmission loss.

If only the lower coupling flange of mechanical impedance $Z_{\mathrm{b}}$ is considered (part of the vehicle body just after the silentblock - usually is $1 / 4$ model used or foundation of the $\mathrm{CGU}$ ), the equation of motion becomes

$$
Z_{\mathrm{b}} \dot{x}_{2}=-Z_{\mathrm{m}}\left(\dot{x}_{2}-\dot{x}_{1}\right)
$$


from which the required transmissibility of vibration or transfer function when the resilient element (silentblock) is loaded by the given force system (pressure) can be then computed

$$
T=\left|\frac{\dot{x}_{2}}{\dot{x}_{1}}\right|=\left|\frac{Z_{\mathrm{m}}}{Z_{\mathrm{b}}+Z_{\mathrm{m}}}\right| .
$$

It is more suitable to directly determine the transmission loss $D$ (in $\mathrm{dB}$ ) of the resilient element which can be calculated from the formula

$$
D=10 \lg \frac{a_{1}^{2}}{a_{2}^{2}} \quad \text { or } \quad D=10 \lg \frac{v_{1}^{2}}{v_{2}^{2}} .
$$

Another method determines the damping capacity $\psi$ of a vibrating system which can be defined as $\psi=\Delta E / E$, where $\Delta E$ represents the energy that is removed from the system per cycle and $E$ denotes the vibration energy stored in the system. The loss factor $\eta$, defined by $\eta=\psi / 2 \pi$, represents the ratio of the energy removed per radian to the stored vibration energy. In most practical situations, loss factors less than 0.2 and 1 can be considered to have a stored energy equal to the total vibration kinetic and potential energy. In general, the various measures of damping are related to each other by

$$
\eta=\frac{\Delta E}{2 \pi E}=\tan \phi .
$$

The phase angle $\phi$ by which the displacement of the mass of the isolator lags means that the excitation force may also be used to characterise the system's damping. For example, at radian frequencies $\omega$ which are far below the system's natural frequency $\tan \phi=k_{i} / k_{0}$ where $k_{i}$ is the imaginary part of complex stiffness

$$
k=k_{0}+\mathrm{j} k_{i} .
$$

The methodology discussed can also be applied to other sources of very low-frequency acoustic vibration as e.g. air-conditioning systems, boiler systems, large low-frequency Diesel engines.

\section{Results and discussion}

The chassis of the vehicle was excited by a deterministic signal for a defined interval of time. From the analysis of the frequency spectra, a strong oscillating frequency was observed, which generated lowfrequency acoustic waves within the cabin (Fig. 2), where the one-third-octave band and FFT analysis in the cabin of the passenger car was carried out. Frequency analysis of the investigated low-frequency region with application of A- and Z-weightings, as presented in Fig. 2, shows the frequency conformity of the results at constant speed. The utilization of the Z-weighting shows direct exposure of the human being to the noise, regardless of the sensitivity of their ears.
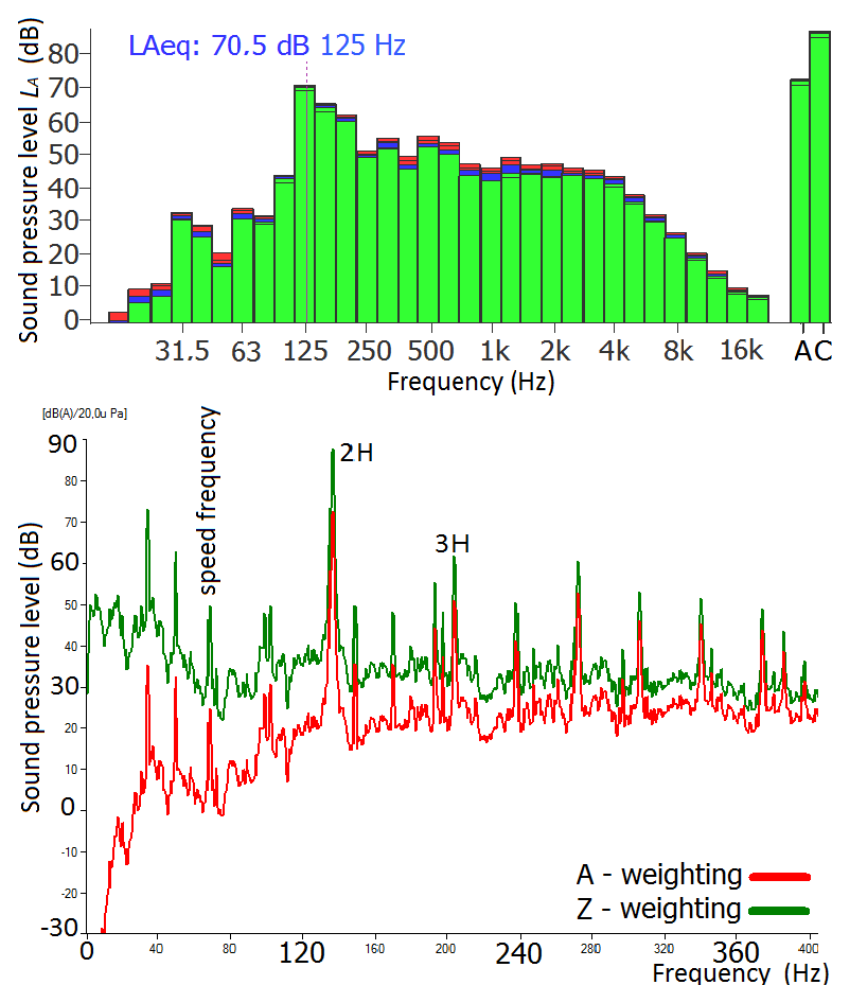

Fig. 2. One-third-octave analysis and FFT analysis of a vehicle body at a given operational speed of its ICE using Z- (upper curve) and A-weightings.

The sensitivity of the human ear at lower frequencies is much lower; therefore so are the measured results, weighted using the A- as well as Cor Z-weightings, are significantly different. Currently, there is a discussion about the evaluation of the low frequency noise of high sound pressure levels, since the A-weighting, which are most often used, do not reflect the correct influences on health and comfort of human beings, in general (DARULA, ZIARAN, 2010a; 2010b; Mirowska, 1995; PAWLACZYK-LUsZCZYNSKA et al., 2010; ZIARAN, 2013a, 2014b; ISO 1996-1:2003].

If the one-third-octave band analysis and FFT analysis are compared, we can state that the determining contribution to A-weighting sound pressure level of the transmitted structural-borne noise to the car cabin has low frequency waves close to $125 \mathrm{~Hz}$. It is known that the sensitivity of the hearing mechanism highly depends on the frequency content of the received sound and at the middle frequency of $31.5 \mathrm{~Hz}$ it is $39.4 \mathrm{~dB}$ lower when compared to the actual sound pressure level (Z-weighting). This indicates that the passengers inside the cabin are exposed to essentially higher lowfrequency sound energy (ZIARAN, 2012) than are the perceptive decibel values and it is confirmed by means of FFT analysis (Fig. 2).

The low-frequencies of the sound and infrasound of different vehicles, building equipments and noise which were analyzed as a whole can be perceived if they 
are sufficiently intense. In general, it has been found that if people are exposed to very low-frequencies of sound and infrasound, they may experience difficulties in performing mental work as well as exhibiting a general sense of discomfort. As intensity increases, dizziness, fatigue, irritation, nausea, and headache may occur (ARGalasova et al., 2013; LEVENTHALL, 2004; ZiARAN, 2008, 2016).

In terms of an internal acoustic environment, it is desirable to know the relationships between the excitation frequency and the revolutions of the motor and the Eigen frequencies of each individual chassis component. Using a modal hammer for modal analysis (ZIARAN, 2013c; 2014a) obtains the Eigen modes (shapes) for the roof and floor pan of the vehicle, which first three and first Eigen (natural) frequencies, respectively, can be seen in Fig. 3 .

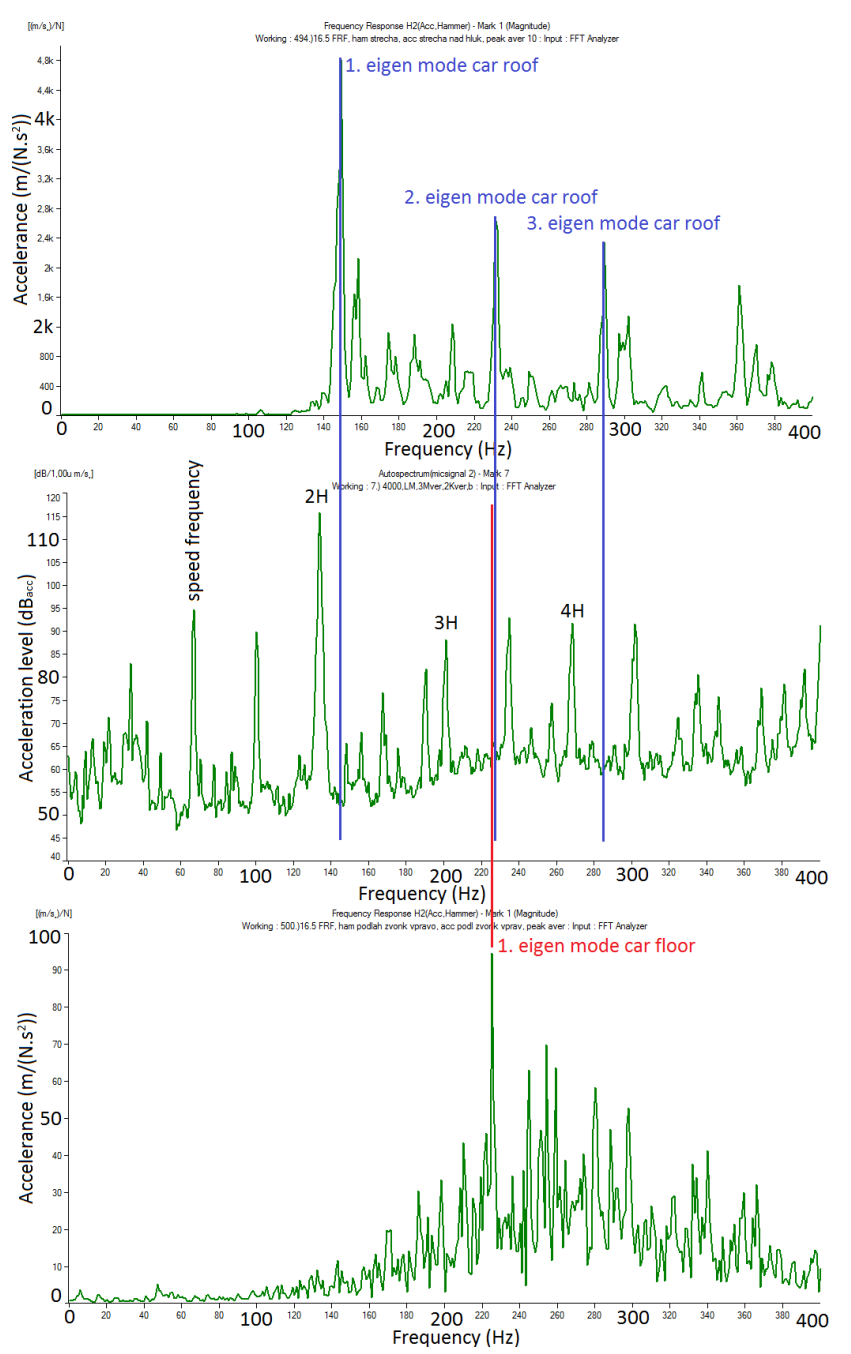

Fig. 3. FFT analysis of the vehicle body beyond the silentblock (in the middle) at a given rpm of the ICE, and Eigen frequencies of the roof (upper) and floor pan (lower) of the same structure as a result of modal analysis.

Comparing the excitation frequency spectra of the vehicles engine with the Eigen frequencies of the ve- hicle roof and deck Eigen modes, some similarities in excitation frequencies and Eigen frequencies can be observed. In this case, such similarity doesn't occur for the defined operational conditions, which is a positive result in terms of generated acoustic energy within the cabin of the vehicle. If the frequencies where to coincide, unwanted resonance would occur, increasing noise levels in the cabin of the vehicle. From the frequency spectra, where the basic rotational and its harmonic frequencies change with respect to speeds, assuming that the natural frequencies remain constant, it is possible to determine at which frequencies resonance will occur for each vehicle component (ORESKY et al., 2012; Ziaran, Chlebo, 2015a).

Comparing the excitation frequency spectra of the vehicles motor with Eigen low-frequencies of the roof and floor pan, the first two Eigen modes were $149 \mathrm{~Hz}$ and $231 \mathrm{~Hz}$ (for the doors, the first two Eigen modes are $70 \mathrm{~Hz}$ and $139 \mathrm{~Hz}$ ), coincidence may be found between the excitation speed frequency and its harmonics and Eigen frequencies at approximately $4000 \mathrm{rpm}$ (Fig. 3). If coinciding frequencies exist in the operational frequencies of the vehicle, it is possible to modify each individual component in order to tune the Eigen frequencies during operation and minimize vibroaccoustic energy within the cabin (ORESKY et al., 2012; ZIARAN, 2014a).

The discussed methodology can be applied for different structures which are used not only in the automobile industry, but also in nuclear power plants or other industry plants and household facilities, provided that certain safety precautions are obeyed (ZIARAN et al., 2013; ZIARAN, 2013b; 2016).

Typical resilient material (elements), used for vibroisolation, consists of an elastic layer, such as rubber for silentblocks, between two rigid surfaces. The forces acting at the two sides of the resilient body stand in a firm relationship with the resulting accelerations and/or velocities at the terminal points. This relationship is frequency dependent and is usually expressed in terms of the direct and transfer impedances of each of the endpoints (Ziaran, 2006).

Once the force-velocity relationship has been determined, either by computation or by measurement, knowledge of the end velocities of the mounted resilient element is sufficient for evaluation of the transmission energy flow throughout the resilient material. Due to inevitable internal losses in the resilient element, the input energy flow must be larger than the output energy flow, as it is confirmed on the gear box silentblock (Fig. 4). Determining frequency components transmitted throughout the silentblock are the frequency and its harmonics. The maximal attenuation of the gear box silentblock is approximately within the entire low frequency range. For the engine block silentblock it is only after $300 \mathrm{~Hz}$ and therefore the energy of lowfrequency waves is transmitted to the body of the vehi- 


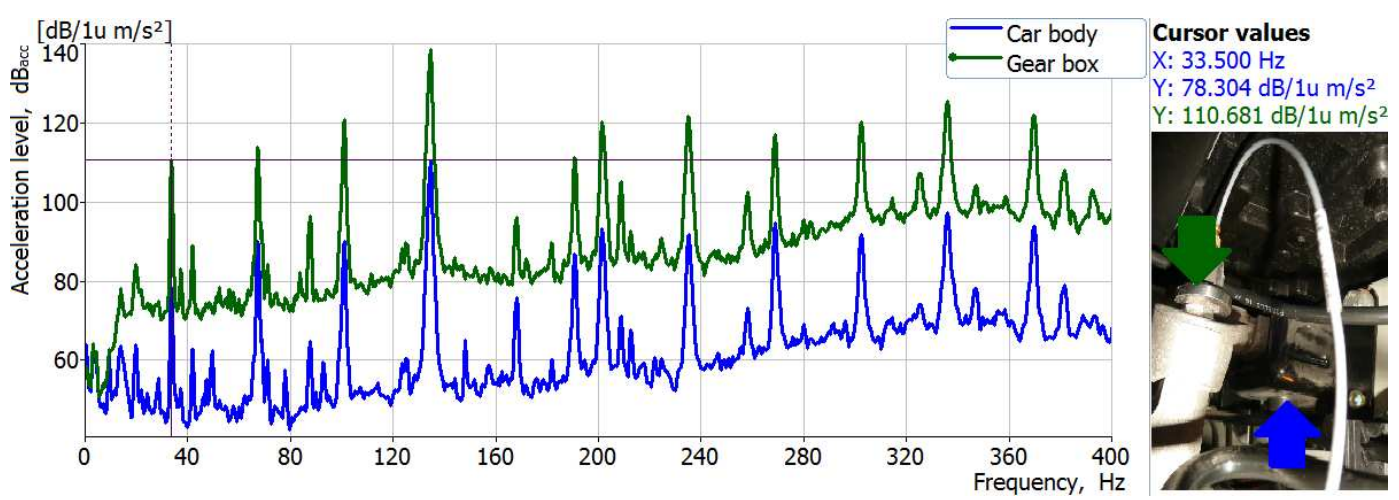

Fig. 4. Frequency spectra at silentblock of the vehicles gear box. In front of the silentblock (up) and beyond the silentblock (down).
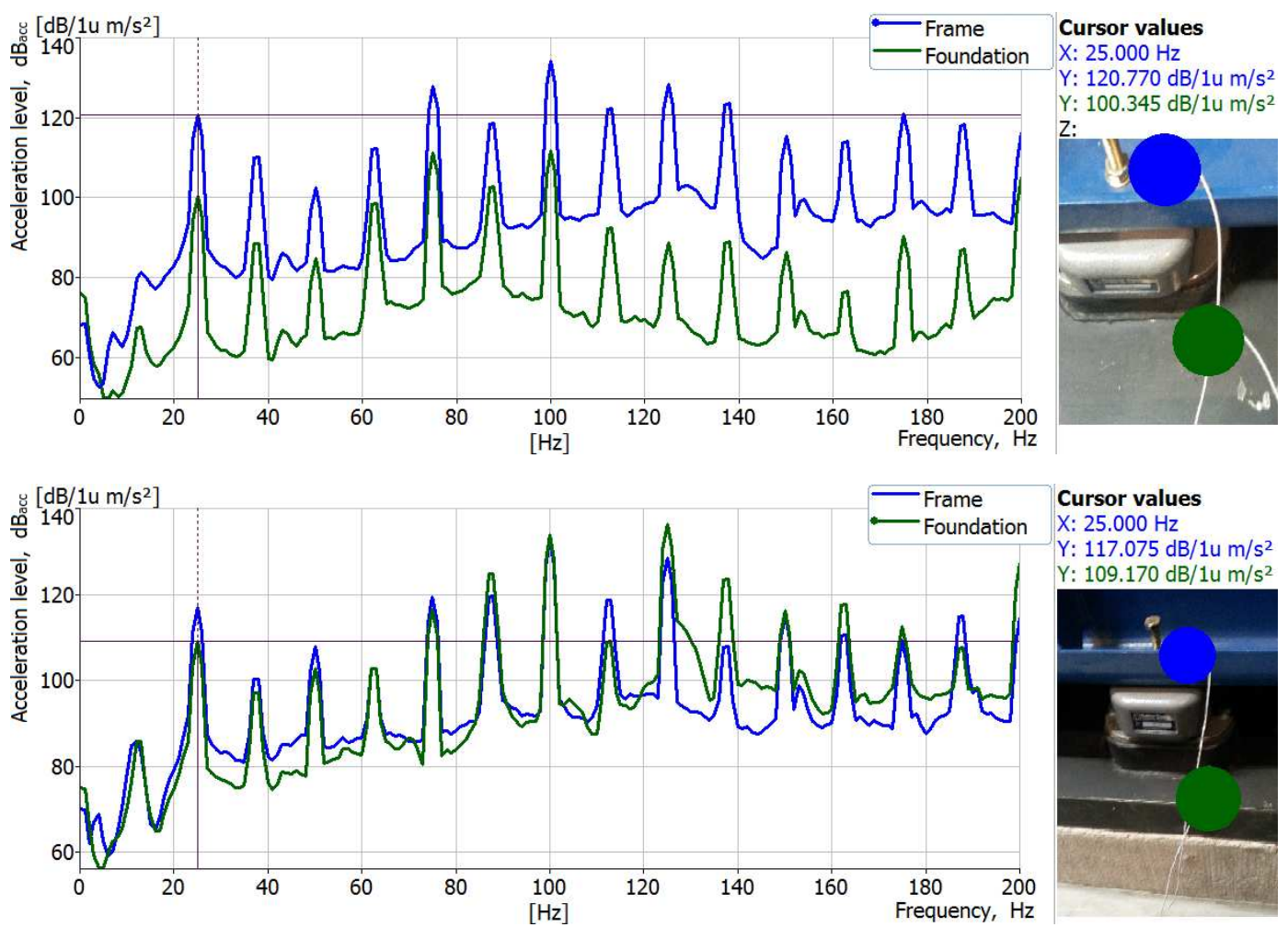

Fig. 5. Transmission vibration energy through two randomly selected CGU silentblocks of the same structure.

cle (Ziaran, Chlebo, 2015b). Similarly, for a cogeneration unit (CGU) silentblock, the attenuation can be different for the same structure of silentblock as is shown thereinafter.

The character of vibration generated during the operating conditions of a CGU is of mechanical nature and can be defined as a continuous spectrum with constant amplitude in the low-frequency domain. High amplitude in the low-frequency audible range is achieved at the speed frequency of $25 \mathrm{~Hz}$ and its harmonics (Fig. 5), that was measured within the surrounding area about $100 \mathrm{~m}$ away (Fig. 6). The transmission of vibration energy through silentblocks also depends on the load and method of attachment

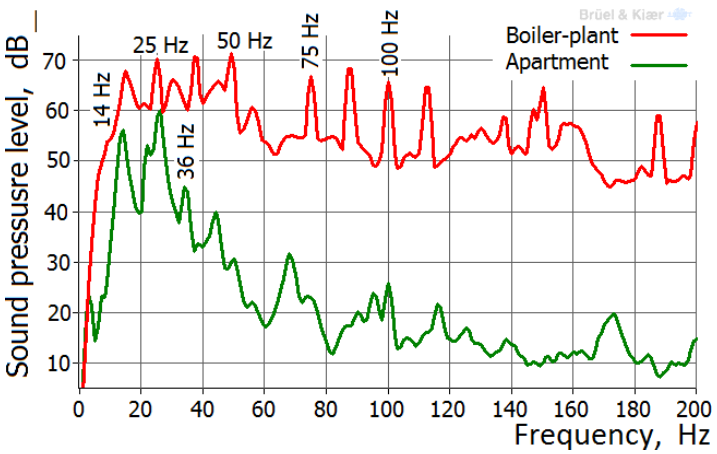

Fig. 6. The frequency spectrum of the noise emitted by a CGU inside (upper curve) and outside a building in an apartment $100 \mathrm{~m}$ away (lower curve). 
(Ziaran, 2006). Frequency response over two randomly selected silentblocks and transmission of the vibration energy to the vibro-isolation building structure is shown in Fig. 5. Comparing the frequency-amplitude characteristics for these silentblocks showed that significant differences in vibration energy attenuation. If the first silentblock performs the function of damping, in the observed attenuation frequency up to $200 \mathrm{~Hz}$ at $21.4 \mathrm{~dB}$ and at the speed of frequency of $25 \mathrm{~Hz}$ it is $20.4 \mathrm{~dB}$, then silentblock 3 in this range amplifies the functions of vibration and hence the attenuation has a negative value $-4.3 \mathrm{~dB}$ (Ziaran, Chlebo, 2015b). Obviously, this silentblock is not set well and there is unwanted contact that results in resonance. It may be stated that unwanted low-frequency waves inside the CGU area are caused by human activities.

The attenuation for each dominant frequency can be determined from the FFT analysis in front and after the resilient element. Assuming only pure translational motion in the direction of its axis, the resilient element (e.g. silentblock, see Fig. 1) is characterized by four impedances $Z_{11}, Z_{22}, Z_{12}, Z_{21}$, where

$$
Z_{\mathrm{ij}}=\left.\frac{F_{\mathrm{i}}}{v_{\mathrm{j}}}\right|_{v_{\mathrm{i}}=0} \quad \text { where } \mathrm{i}, \mathrm{j}=1,2 .
$$

The mechanical impedances $Z_{\mathrm{m}}$ are complex (2), and the frequency dependent quantities are shown in Figs. 4, 5 and 6 . The impedance can be determined by means of force measurements (Ziaran, 2013c). Frequency spectrum of the acceleration measured in front of and after the silentblock of the gear box and at the engine of the CGU for the defined frequency interval is shown in Fig. 4 and Fig. 5, respectively.

Note: The vibration isolators are only effective between structures of relatively large dynamic stiffness on both sides of the isolator and Eq. $(8)_{2}$ represents the intended situation at the receiver side (ZIARAN, 2006; ISO 10846-1:1998], i.e.

$$
k_{2,1}=\left.\frac{F_{2, \text { blocking }}}{x_{1}}\right|_{x_{1} \approx 0},
$$

where $k_{2,1}$ is blocked transfer stiffness and for passive isolators $k_{1,2}=k_{2,1}$ and $x_{1}$ is displacement.

Just as each structure and component of the mechanical systems may resonate, the resilient elements also have Eigen (natural) frequencies which depend on its material properties, boundary conditions and characteristic dimension - thickness of the resilient element. The natural frequencies and mode shapes of an element with both ends built-in can be calculated by

$$
f_{n}=n c_{\mathrm{L}} / 2 L \text {, }
$$

where $c_{L}$ represents the phase speed of the longitudinal wave, $L$ is the thickness of the resilient element and $n$ represents the order of the mode. The thickness of the resilient element according to the frequency spectra is determined using formula (9).

\section{Conclusions}

The theory and given methods can be used for the calculation of the vibration transmissibility of a resilient elements which are used in mechanical and civil engineering. The method is applicable to all materials which behave in a linear way. The methods can be applied for two or more layers forming a sheet. The accuracy (repeatability) of the measured transmissibility is expected to be about $10 \%$. This will depend on the linearity of the sample, the accuracy of the mechanical impedance value $Z_{\mathrm{m}}$ or other part of the body and other measurement parameters (e.g. humidity, temperature, shape of the sample) and the properties of the test material (e.g. ageing, nonlinearity).

The goal of the paper was to carry out the frequency analysis of low-frequency vibration and modal analysis for the passenger car and CGU which generate low-frequency waves that transmit into the cabin area of the passenger vehicle or into the surrounding areas affected by the running of CGU.

Low-frequency waves generated by periodic excitation of rotating machines and/or mechanical systems with reciprocating motion have a negative effect on the mechanical system as a whole as well as effecting any human being within their vicinity as well. The problem of low-frequency vibration sources, transmission and their influence on machines, structures and humans is currently of great interest. Especially, the negative effects on the human body which effect health and psychological disruption when a person is exposed to these frequencies for prolonged periods of time as is shown e.g. in Refs. (Argalasova et al., 2013; BALAZIKova, Sinay, 2012; LeVEnthall, 2004; Ziaran, $2009 ; 2011 ; 2013 d ; 2016)$. Internal noise and especially low-frequency noise has an effect on the comfort of a driver and their passengers which affects the safety and reliability during driving.

On the other hand the energy of low-frequency waves through the construction and maintenance access of CGU is transmitted over relatively long distances and under certain conditions generates standing waves in enclosed areas, which increases the density of sound energy (Ziaran, Chlebo, 2015b; Ziaran, 2016). Investigations have shown that the perception and response of human beings on sound differ considerably at lower-frequencies when compared to mid or high frequencies. Therefore more detail is needed to investigate the sources, transmission and conditions at which low-frequency noise becomes annoying for human beings and negatively impact health (ZIARAN, Chlebo, 2015b).

The effective solution of CGU noise reduction may be possible by means of utilizing vibroisolation on the source structures, and by means of absorptive silencers with the interior reflective elements and their 
proper implementation. The attenuation of the lowfrequency noise depends on the dimensions of a silencing element and can be from $20 \mathrm{~dB}$ up to $60 \mathrm{~dB}$ with maximal length of up to $2.5 \mathrm{~m}$ (ZIARAN, 2016). If the tonal frequency of low-frequency noise is known, then the reflective elements can be exactly situated in the interior of the silencer with maximal effectiveness.

\section{Acknowledgments}

The article is published with the support from the Scientific Grant Agency of the Ministry of Education of the Slovak Republic and the Slovak Academy of Sciences (VEGA) 1/0544/16 and 1/0742/15.

The paper will be presented during the 17 th International Conference on Noise Control 2016.

\section{References}

1. Argalasova L., Lekaviciute J., Jeram S., SevCIKOVA L., JuRkovicova J. (2013), Environmental Noise and Cardiovascular Disease in Adults, Research in Central, Eastern and South-Eastern Europe and Newly Independent States, [in:] Noise Health 2013; 62, $22-31$.

2. Balazikova M., Sinay J. (2012) Implementation of Auditory and Non-auditory Effects of Noise in the Risk Assessment Process in Mechanical Engineering, [in:] Procedia Engineering, No. 48, p. 621-628, ISSN 18777058, http://www.sciencedirect.com/science/journal/ $18777058 / 48$.

3. Darula R., Ziaran S. (2010a), Influence of Specific Noise on Driver's Comfort, [in:] Proceedings of the 5th International Symposium Material - Acoustics - Place 2010, Zvolen, 55-58.

4. Darula R., Ziaran S. (2010b), Noise Exposition in Protected Rooms at Faculty of Mechanical Engineering Slovak University of Technology, [in:] 13th International Conference Mechanical Engineering Bratislava 2010, 30-37.

5. Leventhall H.G. (2004), Low Frequency Noise and Annoyance, Noise Health 2004, 6, 59-72.

6. Mirowska M. (1995), Results of Measurements and Limits Proposal for Low Frequency Noise in the Living Environment, J. Low Frequency Noise Vib., 14, 135141.

7. Oresky J., Ziaran S., Chlebo O. (2012), Transmission of Vibroacoustic Energy Through Body of a Car, Noise and Vibration in Practice: Proc. of the 17th Inter. Acoustic Conference, Bratislava 2012, 65-70.

8. Pawlaczyk-Luszczynska M., Dudarewicz A., SZYMCZAK W., SLIWINSKA-KowAlska M. (2010), Evaluation of Annoyance from Low Frequency Noise under Laboratory Conditions, Noise Health 2010, 12, 166-81.

9. Ziaran S. (2006), Vibration and Acoustic. Noise and Vibration Control in Industry [in Slovak], Monograph, Slovak University of Technology in Bratislava, pp. 332.

10. Ziaran S. (2008), Protection of Human Being against Vibration and Noise [in Slovak], Monograph, Slovak University of Technology in Bratislava, pp. 264.

11. Ziaran S. (2009), Analysis of Annoying Low Frequency Noise Boiler-rooms, [in:] InterNoise2009, Ottawa/Canada.

12. Ziaran S. (2010), Vibro- and Sound-isolation of the Low-frequency Noise of the Building Equipment, InterNoise2010, Lisbon/Portugal, 3263-3272(10).

13. Ziaran S. (2011), Effects of Low-frequency Noise in Closed Space on the Human, InterNoise2011, Osaka/Japan.

14. Ziaran S. (2012), Low Frequency Noise from the Open Window in a Moving Car and its Effect on Human Beings, InterNoise2012, New York/USA.

15. Ziaran S. (2013a), Low Frequency Noise and its Assessment and Evaluation, Archives of Acoustics, 38, 2, 253-270.

16. Ziaran S. (2013b), Low Frequency Vibration and Noise Generated by Seismic Sources and their Effects on Surroundings, InterNoise2013, Innsbruck/Austria.

17. Ziaran S. (2013c), Technical Diagnostics Scientific [in Slovak], Monograph, Issued by Slovak University of Technology Bratislava, pp. 332.

18. Ziaran S. (2013d), Potential Health Effects of Standing Waves Generated by Low Frequency Noise, Noise Health 2013, 15, 237-45.

19. Ziaran S. (2014a), Using Frequency and Modal Analysis to Attenuate Low Frequency Waves, InterNoise2014, Melbourne/Australia.

20. Ziaran S. (2014b), The Assessment and Evaluation of Low Frequency Noise Near Region of Infrasound, Noise Health 2014, 16, 10-7.

21. Ziaran S. (2016), Low Frequency Noise and Vibration [in Slovak], Monograph, Issued by STU Bratislava 2016 , pp. 316.

22. Ziaran S., Chlebo O. (2015a), Transmission of Vibroacoustic Energy Through the Structure of a Car Body into the Protected Area and its Control, EuroNoise 2015, Maastricht, EAA-NAG-ABAV, p. 491496.

23. Ziaran S., Chlebo O. (2015b), Exposure to Lowfrequency Noise from the Boiler-plant to the Apartment Svabinskeho Street [in Slovak], Research Report FME STU Bratislava. 
24. Ziaran S., Musil M., Cekan M., Chlebo O. (2013), Analysis of Seismic Waves Generated by Blasting Operations and their Response on Buildings, International Journal of Environmental, Earth Science and Engineering, $\mathbf{7}, 11$.

25. ISO 1996-1:2003 Acoustics. Description, Measurement and Assessment of Environmental Noise. Part 1: Basic
Quantities and Assessment Procedures, ISO copyright office Case postale 56 • CH-1211 Geneva 20.

26. ISO 10846-1:1998 Acoustics and Vibration. Laboratory Measurement of Vibro-acoustic Transfer Properties of Resilient Elements. Part 1: Principles and Guidelines, ISO copyright office Case postale $56 \bullet \mathrm{CH}-1211$ Geneva 20 . 